\title{
Monte-Carlo simulation of Ising droplets in correlated site-bond percolation
}

\author{
D. Stauffer (*) \\ Département de Physique des Systèmes Désordonnés, Université de Provence, \\ Centre Saint-Jérôme, 13397 Marseille Cedex 13, France
}

(Reçu le 3 décembre 1980, accepté le 19 janvier 1981)

\begin{abstract}
Résumé. - On étudie par ordinateur la définition de Coniglio et Klein pour les gouttes du modèle Ising, sur les réseaux carré et cubique simple. Nos résultats sont compatibles avec leur prédiction que les gouttes divergent à $T_{\mathrm{c}}$ : pour les réseaux $16 \times 16 \times 17$, la divergence est située à $T / T_{\mathrm{c}}=1,02$. Au-dessus de $T_{\mathrm{c}}$ à trois dimensions et champ magnétique nul, en fonction de la concentration des liens actifs, on trouve à peu près le même exposant critique $v=0,9$ que pour la percolation aléatoire ; mais le taux de percolation est doublé.

Abstract. - The definition of droplets in the Ising model by Coniglio and Klein is investigated numerically on square and simple cubic lattices. Our data are consistent with their prediction that the droplets diverge at $T_{\mathrm{c}}$ : for $16 \times 16 \times 17$ lattices, the divergence occurs at $T / T_{\mathrm{c}}=1.02$. Above $T_{\mathrm{c}}$ in three dimensions and zero magnetic field, as a function of the concentration of active bonds, we find about the same critical exponent $v=0.9$ as for random percolation; but the percolation threshold is twice as large.
\end{abstract}

1. Introduction. - The description of collective phenomena, in particular at phase transitions, by a model of noninteracting droplets has a long tradition in statistical physics, going back to the Mayer cluster theory for equilibrium properties and to nucleation theory for a nonequilibrium phenomenon. Percolation turned out to be a problem where by definition this method works particularly well [1]. Also in the Ising model (or lattice gas) one may define a cluster by a group of up spins, connected by ferromagnetic exchange energies $J$ and surrounded by a background of down spins. Unfortunately, in three dimensions these Ising clusters diverge at a temperature $T=0.95 T_{\mathrm{c}}$ below the critical temperature [2], and in two dimensions where the position of the critical point is correct the exponents are not [3]. Thus the above cluster definition is not suitable. Some attempts have been made [4] to circumvent these difficulties by relating phenomenologically the geometrical cluster size $s$ to an effective cluster size $\propto s^{y}$, with a suitable exponent $y$.

In a different, more microscopic approach of Coniglio and Klein [5] which we adopt here, loosely connected clusters are split into different droplets by a probabilistic approach : A bond between two neighbouring up spins is regarded as active (in the

$\left(^{*}\right)$ Permanent address : Institut für Theoretische Physik, Universität, 5000 Köln 41, West Germany. sense of the droplet definition) only with probability $p_{\mathrm{B}}$, and remains inactive with probability $1-p_{\mathrm{B}}$. The concentration of active bonds is given by [5]

$$
p_{\mathrm{B}}=1-\exp (-2 J / k T)
$$

an expression already known from the KasteleynFortuin theorem (P. W. Kasteleyn, C. M. Fortuin, J. Phys. Soc. Japan, Suppl. 26 (1969) 11). The Ising exchange interaction $J$ is called $\tilde{K}(=K / 4$, not $=4 K)$ in reference [5] and determines the critical temperature $T_{\mathrm{c}}$ through $2 J / k T_{\mathrm{c}}=\ln (1+\sqrt{2})=0.881$ in two and $=0.454$ in three dimensions. At infinite temperatures no bond is active according to equation (1) whereas at $T=0$ all bonds between up spins are active.

Coniglio and Klein call Ising droplets the structures of up spins connected by active bonds between neighbouring up spins as opposed to the larger Ising clusters defined as usual as groups of neighbouring up spins independent of the status of the bonds connecting them. Figure 1 shows an example of how a large Ising cluster at $T / T_{\mathrm{c}}=3$ is broken up into smaller droplets. (Note that the magnetic properties are not changed at all due to the introduction of inactive bonds; $p_{\mathrm{B}}$ only changes the geometry of the droplets, not the exchange interactions which are always present.) Coniglio and Klein suggest these droplets, defined via equation (1), as good candidates for a droplet description of the Ising model near its critical point. The 


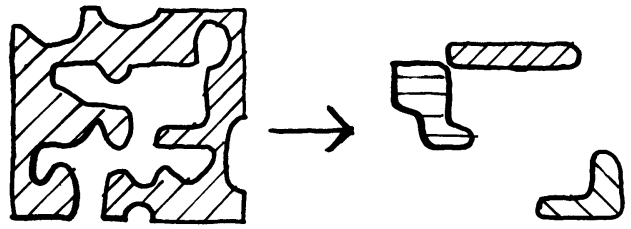

Fig. 1. - Breakup of a large Ising cluster (left) into smaller Ising droplets (right), in a $10 \times 11$ square lattice at $T / T_{\mathrm{c}}=3$. In the right part, only about $1 / 4$ of all bonds between nearest-neighbour up spins are regarded as active according to equation (1). Only droplets with more than three sites are shown.

present work tries to check if indeed these droplets diverge at $T_{\mathrm{c}}$, as predicted theoretically [5]. Except where stated otherwise our results hold for three dimensions in zero magnetic field.

2. Numerical techniques. - Our Monte-Carlo simulation used nearest-neighbour lattices with

$$
L \times L \times(L+1)
$$

sites in three and $L \times(L+1)$ sites in two dimensions. (The additional site in the last direction was used to preserve self-matching in two dimensions [6] to get the bond percolation thresholds more accurately.) $L$ varied from 5 to 50 in two and from 3 to 16 in three dimensions; about 20 different temperatures were investigated. Periodic boundary conditions were used for the magnetic interaction [7] but free boundaries for the connectivity properties [8]. For $L=16$ in three dimensions we used multi-spin coding techniques [9], i.e. we stored 8 spin variables in each 32-bit computer word. This technique reduced the computing time for the relaxation of the magnetization by a factor of about two, as opposed to the factor of more than three observed [9] for 60-bit words containing 20 spins. (With 10 spins in a 32-bit word nearly all time advantage was lost.) For infinite fields we confirmed within $10^{-2}$ the known bond percolation thresholds 0.50 and 0.25 for square and cubic lattice. In general, we start from a configuration with all spins down, i.e. no clusters and no droplets at the beginning. We then looked at the creation of clusters and droplets in intervals of 25 Monte-Carlo steps per spin (MCS); away from $T_{\mathrm{c}}$ the interval was only $10 \mathrm{MCS}$. We averaged over equilibrium properties from about $10^{2}$ to about $10^{3}$ MCS. After each time interval of 25 or 10 MCS we checked if the Ising cluster percolates in the direction where $L+1$ spins are present; this problem corresponds to interacting percolation [10]. If the Ising cluster does not percolate, none of the corresponding droplets can percolate, and we go to the next time interval. If the cluster percolates we check with 5 or 10 different sets of random numbers if also an Ising droplet, for the given concentration $p_{\mathrm{B}}$ of active bonds between up spins, percolates in the same direction; this question is the correlated sitebond percolation problem $[11,3]$.
3. Percolation thresholds. - If the bond concentration $p_{\mathrm{B}}$ is given by equation (1), then in general no percolating droplet was found. Far below $T_{\mathrm{c}}$ in our samples the concentration of up spins is so low that clusters cannot percolate. Far above $T_{\mathrm{c}}$ there is always a percolating clusters; but now $p_{\mathrm{B}}$ is so low that the droplets cannot percolate. For $L=16$, only at $T / T_{\mathrm{c}}=1.02 \mathrm{did}$ we find one example where droplet percolation occurred at a concentration of active bonds at or below the value given by equation (1); for smaller samples such events occurred more often. This is our first indication that for infinite samples the droplets percolate at $T_{\mathrm{c}}$.

For a more quantitative treatment we generalized the model by allowing $p_{\mathrm{B}}$ to be larger than before by an excess $\Delta p$ :

$$
p_{\mathrm{B}}=\Delta p+1-\exp (-2 J / k T) .
$$

Thus $p_{\mathrm{B}}$ is regarded as a variable independent of temperature and field. After every time interval of 25 or 10 MCS we make 10 different iterations [12] to find out what the actual percolation threshold $p_{\mathrm{Bc}}$ is for this particular spin configuration. We averaged over all configurations and iterations in equilibrium to determine the average threshold $\left\langle p_{\mathrm{Bc}}\right\rangle$ and its fluctuation

$$
W^{2}=\left\langle p_{\mathrm{Bc}}^{2}\right\rangle-\left\langle p_{\mathrm{Bc}}\right\rangle^{2}
$$

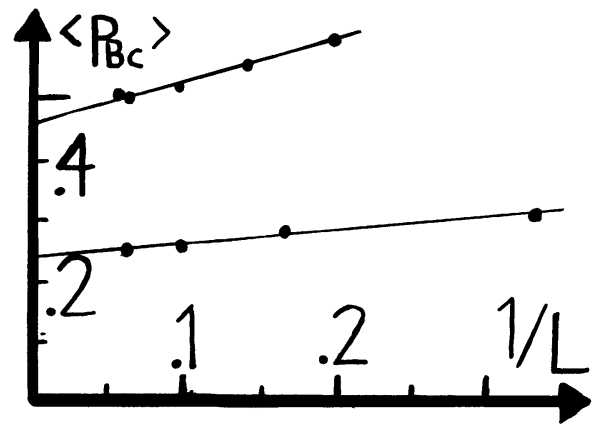

Fig. 2. - Size dependence of the average percolation threshold for correlated site-bond percolation at $T / T_{\mathrm{c}}=1.5$ (top) and for random bond percolation (bottom) in the simple cubic lattice.

Figure 2 gives a plot of the average threshold versus the reciprocal system size; the intercept is an extrapolation for $\left\langle p_{\mathrm{Bc}}\right\rangle$ in the infinite system. In figure 3 we give these extrapolated thresholds, together with the excess concentration

$$
\Delta p_{\mathrm{c}}=\left\langle p_{\mathrm{Bc}}\right\rangle-1+\exp (-2 J / k T) .
$$

We see that our data for $\Delta p_{\mathrm{c}}$ extrapolate to zero at or close to the critical temperature; i.e. at or near the critical point the droplets given by equation (1) diverge.

A more accurate estimate of the temperature, at which the droplets become infinitely large, is obtained if we start the Monte-Carlo simulation with all spins 


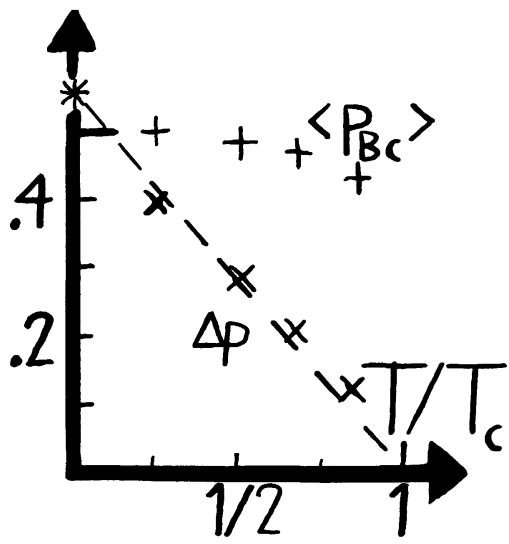

Fig. 3. - Temperature dependence of the average percolation threshold $(+)$ and of the excess concentration $\Delta p$ defined by equation (2) ( $x$ ) for a simple cubic lattice, extrapolated to infinite size $L$ by an $L^{-1.2}$-law (cf. last figure). The dashed line through the data for the excess concentration suggests that the latter vanishes at the critical point, as predicted by Coniglio and Klein.

up instead of all spins down; that means at the beginning we have one infinite cluster, and later this cluster breaks up due to thermal fluctuations. Then below $T_{\mathrm{c}}$ and, for finite systems, slightly above $T_{\mathrm{c}}$ the spontaneous magnetization $M$ is positive, the concentration $x$ of up spins, with

$$
x=(1+M) / 2
$$

is far above the cluster percolation threshold (which is the site percolation threshold $\mathbf{0 . 3 1}$ for random site percolation on the simple cubic lattice), and we can for all temperatures find a droplet percolation threshold $\left\langle p_{\mathrm{Bc}}\right\rangle$. This percolation curve $\left\langle p_{\mathrm{Bc}}\right\rangle(T)$ meets the concentration $p_{\mathrm{B}}$ of equation (1) for $L=16$ at $T / T_{\mathrm{c}}=1.02$ (with a statistical error of less than 0.01), which happens also to be the temperature where, as mentioned above, we observed a droplet percolating for the predicted concentration of equation (1). We plan to make simulations for $L=40$ in order to check if this effective critical temperature of $1.02 T_{\mathrm{c}}$ at $L=16$ shifts towards the true critical temperature $T_{\mathrm{c}}$ for $L \rightarrow \infty$. Figure 4 shows the percolation curves for the two cases where all spins are initially up and all spins are initially down; only the first curve crosses the $p_{\mathrm{B}}(T)$ of equation (1), whereas the other bends strongly in order to reach $\left\langle p_{\mathrm{Bc}}\right\rangle=1$ at the temperature of about $0.95 T_{\mathrm{c}}$, where cluster percolation has its threshold [2]. For $T / T_{\mathrm{c}}$ larger than about 1.1 at $L=16$ the two curves coincide since now the spontaneous magnetization has vanished.

The same data give a much simpler curve, figure 5, if $\left\langle p_{\mathrm{Bc}}\right\rangle$ is plotted not against temperature but against the concentration $x=(1+M) / 2$ of up spins, for temperatures at or below $T_{\mathrm{c}}$. Then a single and simple curve is obtained, similar to random site-bond percolation [13]. The complicated behaviour in the preceding figure 4 is thus due to the complicated behaviour of the spontaneous magnetization as function of sys-

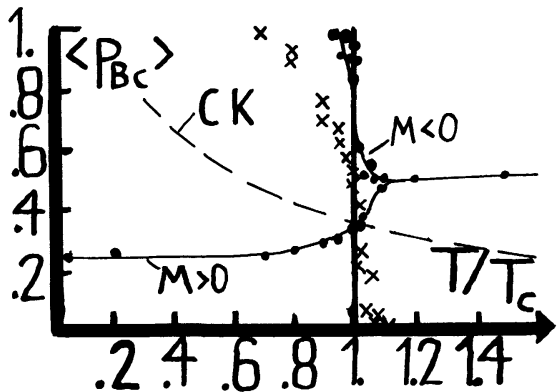

Fig. 4. - Temperature dependence of the average percolation threshold (dots) and the spontaneous magnetization (crosses) for three dimensions with $L=16$. The dashed line is the prediction of equation (1) and is supposed to meet the lines for the percolation threshold at $T=T_{\mathrm{c}}$, for infinite systems. The solid lines are guides to the eye.

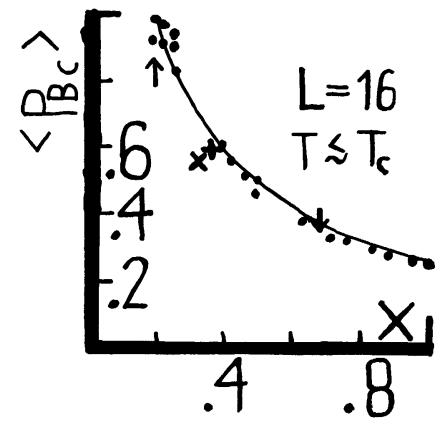

Fig. 5. - Variation of the average percolation threshold with concentration $x=(1+M) / 2$ (same data as in figure 4), at $L=16$ below and near $T_{\mathrm{c}}$ on the coexistence curve. $\uparrow$ gives the limit $\left\langle p_{\mathrm{Bc}}\right\rangle=1$ from reference [2], with $L=30$; $\downarrow$ marks the position where the prediction of equation (1) crosses the Monte-Carlo phase transition line $(M>0)$ of the preceding figure, at $T / T_{\mathrm{c}}=1.02$. Two points for $L=10(+)$ and $5(\times)$ are also given.

tem size and temperature, and not due to complicated percolation effects. This simplicity of figure 5 , as compared with figure 4 , also suggests that the droplets of the correlated site-bond percolating problem describe the magnetic behaviour of the Ising model and not some peculiarities of their connectivity properties. Figure 5 also confirmes at $\left\langle p_{\mathrm{Bc}}\right\rangle=1$, i.e. for Ising clusters, the percolation threshold at $x=0.2$ found already by Müller-Krumbhaar [2] for $L=30$.

4. A critical exponent. - According to finite-size scaling and real space renormalization theory $[6,12]$ the width $W$ of the threshold distribution, equation (3), should vary as $L^{-1 / v}$, where $v$ is the exponent for the connectivity length (typical droplet radius). Figure 6 indicates that the exponent for our correlated sitebond percolation problem is roughly the same as that for random site or bond percolation $[12,14]$ in the simple cubic lattice. The prefactor is larger, however, since we now have thermal fluctuations in addition to fluctuations in the connectivity properties. And, of course, the percolation threshold is higher than for random bond percolation since many sites are missing ; 


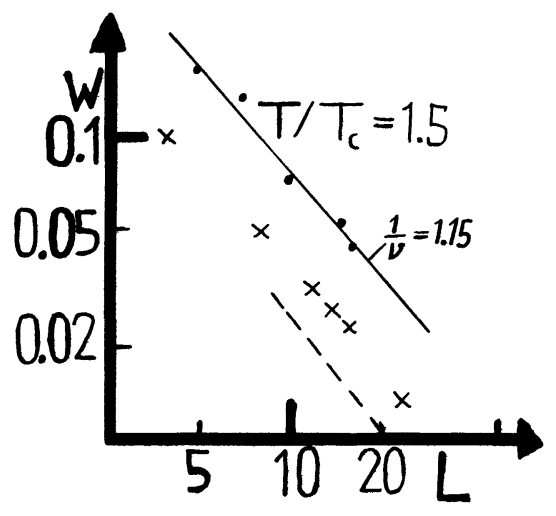

Fig. 6. - Log-log plot of the width of the percolation threshold distribution as a function of lattice size $L$ at $T / T_{\mathrm{c}}=1.5$ (dots). The crosses are results of Levinshtein $e t$ al. for random site percolation, the dashed line those of Kirkpatrick for random bond percolation up to $L=80$. All data have roughly the same slope $1 / v$, in agreement with the universality hypothesis.

above $T_{\mathrm{c}}$ the threshold is about doubled. The present model has been applied to gelation [11] to describe the interplay of phase separation and chemical binding in solutions of branching polymers. Our result then means : for conditions where no phase separation occurs the gel point is shifted to much higher conversion factors $\left\langle p_{\mathrm{Bc}}\right\rangle$ but the critical exponent at this shifted gel point is the same as if no concentration fluctuations would have occurred.

5. Two dimensions. - The square lattice behaves qualitatively different from the simple cubic lattice [5]. At zero field and infinite temperature the Ising clusters are those of random site percolation which has a threshold of 0.31 in three and 0.59 in two dimensions. Thus in a large sample at zero field and far above $T_{\mathrm{c}}$ percolation of clusters occurs always in three and never in two dimensions, and we need a positive magnetization to study droplet percolation. In the presence of a positive magnetic field, at $T / T_{\mathrm{c}}=2$ for
$L=5,20$ and 50 we find a percolation curve, $\left\langle p_{\mathrm{Bc}}\right\rangle$ as a function of concentration $x$, which extends nearly linearly from the exact $\left\langle p_{\mathrm{Bc}}\right\rangle=1 / 2$ at $x=1$ (infinite field) to $\left\langle p_{\mathrm{Bc}}\right\rangle=0$ at $x \simeq 0.5$ (zero or very small field; $|M|<0.1$. To study the behaviour below and at $T_{\mathrm{c}}$ one should start with all spins up in order to get the phase transition curve; but we have not yet done that. In this sense our incomplete results in two dimensions do neither confirm nor contradict the phase diagram postulated in reference [5].

6. Conclusion. - Our three-dimensional results confirm within a few percent that the Coniglio-Klein droplets indeed diverge at the critical temperature of the Ising model. Our system size was not yet large enough to distinguish reliably between the onset of cluster percolation and of droplet percolation. We plan in the future to use a larger and faster computer than the IBM 1130 and CII Iris 80 employed for the present study. One should also look at the droplet size distribution and its critical exponents, for the concentration of active bonds given by equation (1). And the two-dimensional system has to be studied more completely, as well as the field dependence in three dimensions.

Acknowledgments. - We thank Dr. J. Roussenq and many other members of this university for the introduction into the mysteries of its computers, and Dr. E. Guyon for comments on a preliminary version of the manuscript.

Note added in proof. - For the average number $n_{s}$ of droplets containing $s$ occupied sites each, F. Delyon and B. Souillard (private communication, Dec. 1980) proved for $s \rightarrow \infty$, arbitrary $p_{\mathrm{B}}$ and arbitrary magnetic field, in all dimensions $d$, that $n_{s} \geqslant \exp \left(-\right.$ const. $\left.s^{1-1 / d}\right)$ provided that an infinite droplet is present in the system or in one of its two coexisting phases.
[1] Stauffer, D., Phys. Rep. 54 (1979) 1 ; Essam, J. W., Rep. Prog. Phys. 43 (1980) 833.

[2] Müller-KrumbhaAr, H., Phys. Lett. 50A (1974) 27.

[3] Coniglio, A., Nappi, C., Perrugi, F., Russo, L., J. Phys. A 10 (1977) 205;

Sykes, M. F., Gaunt, D. S., J. Phys. A 9 (1976) 2131.

[4] Binder, K., Ann. Phys. (NY) 98 (1976) 390; Stauffer, D., J. Phys. A 10 (1977) L-71;

NAGAO, N., Osaka University preprint.

[5] Coniglio, A., Klein, W., J. Phys. A 13 (1980) 2775.

[6] RousSENQ, thesis, Université de Provence (1980).

[7] Landau, D. P., Phys. Rev. B 13 (1976) 2997 and 14 (1977) 255.

[8] Heermann, D. W., Stauffer, D., Z. Phys. B 40 (1980) 133.

[9] Zorn, R., HerrmanN, H. J., RebBi, C., preprint for Comp. Phys. Comm., with earlier literature on multi-spin coding.

[10] Stoll, E., Binder, K., Schneider, T., Phys. Rev. B 6 (1972) 2777.
[11] Coniglio, A., Stanley, H. E., Klein, W., Phys. Rev. Lett. 42 (1979) 518.

[12] Levinshtein, M. E., ShklovskiI, B. I., ShUR, M. S., EFros, A. L., Sov. Phys. JETP 42 (1976) 197 ;

Reynolds, P. J., Stanley, H. E., Klein, W., Phys. Rev. B 21 (1980) 1223 ;

Eschbach, P. D., STauffer, D., Herrmann, H. J., Phys. Rev. B 23 (1981) 422.

[13] Nakanishi, H., Reynolds, P. J., Phys. Lett. 71A (1979) 252; Hoshen, J., Klymko, P., Kopelman, R., J. Stat. Phys. 21 (1979) 583 ;

Agrawal, P., Redner, S., Reynolds, P. J., Stanley, H. E., J. Phys. A 12 (1979) 2073;

Brown, E., Essam, J. W., Place, C. M., J. Phys. C 8 (1975) 321.

[14] KiRKPATRICK, S., in : Ill Condensed Matter, edited by R. Balian (North Holland Publishing Company, Amsterdam) 1979. 